\title{
First-principles study of alkali-metal intercalation in disordered carbon anode materials
}

Received 00th January 20xx, Accepted 00th January 20xx DOI: $10.1039 / x 0 x x 00000 x$

\begin{abstract}
Jian-Xing Huang, ${ }^{a}$ Gábor Csányi, ${ }^{b}$ Jin-Bao Zhao, ${ }^{a, *}$ Jun Cheng ${ }^{a, *}$ and Volker L. Deringer ${ }^{b, c, *}$
Graphite and non-graphitising ("hard") carbons are important anode materials for battery technologies. The electrochemical intercalation of alkali metals in graphite has been widely studied by first-principles density-functional theory (DFT). However, similar investigations of disordered "hard" and nanoporous carbons have been challenging due to the structural complexity involved. Here, we combine DFT with machine-learning (ML) methods to study the intercalation of alkali metal (Li, Na, K) atoms in model carbon systems over a range of densities and degrees of disorder. We use a stochastic approach to compute voltage-filling profiles, studying the three metal species side-by-side, and we analyse the ionic charges of metal atoms as a function of filling. Our study provides atomic-scale insight into the intercalation of all three alkali metals that are relevant to batteries, and it thereby makes a key step towards the DFT/ML-driven modelling of energy materials.
\end{abstract}

\section{Introduction}

Graphite and related carbonaceous materials can reversibly intercalate metal atoms to store electrochemical energy in batteries. ${ }^{1-5}$ The currently most widespread application is in Li-ion batteries (LIBS), where the formation of well-ordered intercalation compounds $\mathrm{LiC}_{12}$ and $\mathrm{LiC}_{6}$ and the "staging" mechanism have been studied. ${ }^{1}$ Interestingly, $\mathrm{Na}$ atoms do not intercalate into ideal graphite, ${ }^{6}$ while the larger $\mathrm{K}$ atoms do. ${ }^{7-9}$

First-principles computations, most widely based on density-functional theory (DFT), are a central part of modern battery research: they give atomic-scale insight into the various mechanisms at play, and today they can help to find new candidate materials for improved batteries (e.g., with increased capacity). ${ }^{10-12}$ The $\mathrm{LiC}_{6}$ intercalation compound has been studied by DFT methods for many years; ${ }^{13-15}$ a recent work explored a large space of possible structures using a DFT-parameterised model. ${ }^{16}$ Potassium intercalation phases including the $\mathrm{KC}_{8} \mathrm{com}$ pound have likewise been explored by DFT. ${ }^{17-20}$

There have also been important initial steps towards the computational modelling of defective carbon structures, beyond pristine graphite and graphene. For example, the effect of vacancies on metal atom adsorption on graphene was studied, as was the intercalation of $\mathrm{Na}$ in defective graphite. ${ }^{21-23} \mathrm{How}$ ever, more complicated systems (especially, "hard" and nongraphitising carbons) require even much larger structural models to be realistically described. Amorphous carbon ${ }^{24}$ and silicon anodes $^{25}$ have been looked at with model DFT computations. Larger model system sizes are principally accessible to empirically fitted force fields, ${ }^{26-30}$ and indeed ReaxFF type force fields have been described for $\mathrm{Li}-\mathrm{C}$ phases $^{31}$ and $\mathrm{Na}$ intercalation; ${ }^{32}$ however, these methods are necessarily limited by the fixed functional form of the interatomic potential, and they allow no insight into the electronic structure.

In an initial communication, ${ }^{33}$ we have recently argued that carbon-based energy materials can be accurately described in atomistic simulations with a machine learning $(\mathrm{ML})$ based interatomic potential for carbon. ${ }^{34}$ Such $\mathrm{ML}$ potentials perform a high-dimensional fit to complex potential energy surfaces and allow for materials modelling with close-to-DFT accuracy but require only a small fraction of the cost; ${ }^{35-39}$ we recently demonstrated the large synergy between ML and DFT methods for functionalised amorphous carbon materials. ${ }^{40,41}$ Generating the carbon structure in an ML-driven simulation bypasses the need for costly quantum-mechanical computations at runtime, making disordered anode materials such as "hard" carbons accessible to extended molecular-dynamics (MD) runs; in turn, once the carbon structures have been generated, they can be further treated with DFT to study chemical reactivity. ${ }^{33}$ Beyond carbon, recent neural-network-type $\mathrm{ML}$ potentials for $\mathrm{Li}-\mathrm{Si}$ phases ${ }^{42,43}$ attest to the usefulness of such simulation tools. Even further, ML methods are beginning to be used in several other areas of energy materials research, such as the screening for suitable compositions. ${ }^{44,45}$

\footnotetext{
a. State Key Laboratory of Physical Chemistry of Solid Surfaces, College of Chemistry and Chemical Engineering, Xiamen University, Xiamen 361005, China

b. Department of Engineering, University of Cambridge, Cambridge CB2 1PZ, UK

c. Department of Chemistry, University of Cambridge, Cambridge CB2 1EW, UK

*E-mail: jbzhao@xmu.edu.cn (J.-B.Z.); chengjun@xmu.edu.cn (J.C.); vld24@cam.ac.uk (V.L.D.)
}

Electronic Supplementary Information (ESI) available: supplementary results and structural data. See DOI: 10.1039/x0xx00000x 
a

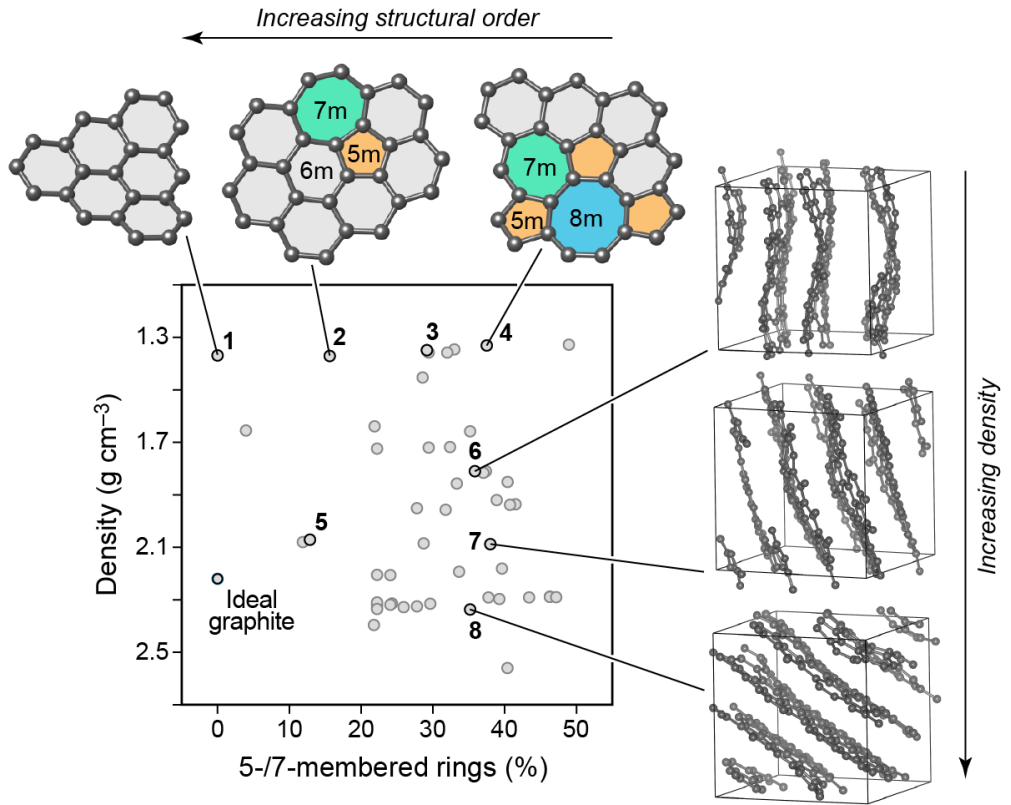

b $\begin{array}{lcc}\begin{array}{c}\text { Select carbon } \\ \text { structure (1-8) }\end{array} & \begin{array}{c}\text { Randomised } \\ \text { cells at filling } x_{1}\end{array} & \begin{array}{c}\text { Randomised } \\ \text { cells at filling } x_{n}\end{array}\end{array}$
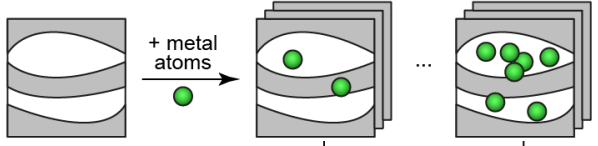

$\downarrow$ DFT relaxation $\downarrow$

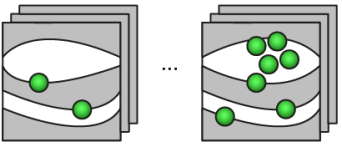

$\downarrow$ DFT total energy $\downarrow$

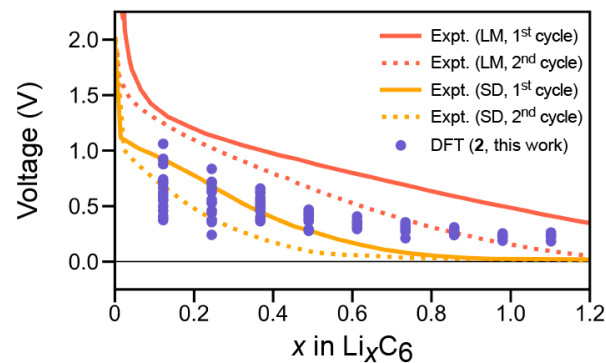

Fig. 1 A computational approach for modelling metal intercalation in disordered carbon materials. (a) A library of graphitic-like structural models that form the basis for our work. Initially, 50 independent simulation cells (with $\approx 200$ atoms each) were created, starting from fully disordered amorphous precursors and inducing graphitisation by thermal annealing. We characterise the resulting structures in the parameter space of structural order, for which the ring statistics serve as proxy (horizontal axis), and mass density (vertical axis). In this plot, representative structures (1-8) are highlighted, and these form the basis for the study reported in the remainder of this paper. (b) Schematic overview of the protocol we use to obtain voltage-filling relations; see text for details. (c) Example of the resulting data (blue), here shown for Li intercalation in $\mathbf{2}$, and compared to experimental data by Legrain, Manzhos, and co-workers ("LM"; red; taken from ref. 24) and by Stevens and Dahn ("SD"; orange; taken from ref. 46), respectively.

Here, we report on a comprehensive computational survey of alkali-metal $(M=\mathrm{Li}, \mathrm{Na}$, and $\mathrm{K})$ intercalation in structural models of $\mathrm{sp}^{2}$-rich disordered carbon materials that are relevant to battery research. We generate an ensemble of gradually ordered carbon structures by ML-driven high-temperature annealing simulations, and then insert metal atoms whose local structures, energetics (hence, voltage profiles), and charge states are studied by DFT. Our work outlines a general strategy for modelling metal intercalation in disordered materials and provides new atomistic insight into carbonaceous anodes beyond ideal and defective graphite.

\section{Methodology}

\section{Disordered carbon structures from GAP-driven simulations}

A common approach for modelling disordered carbons is to start with an ideal graphite or graphene supercell in which one or a few structural defects are then introduced. 47,48 In contrast, we here begin with fully disordered precursors that are partially annealed in $\mathrm{MD}$ runs, forming $\mathrm{sp}^{2}$-rich graphite-like regions as the simulation progresses (typically, over hundreds of thousands of time steps). The approach has been pioneered by Marks and co-workers, ${ }^{26,49-51}$ using the environment-dependent interatomic potential (EDIP), 52 and we used a comparable protocol with an ML interatomic potential in an initial proof-ofconcept study. ${ }^{33}$

Specifically, $a-C$ precursors were generated in melt-quench simulations using the Gaussian Approximation Potential (GAP) framework ${ }^{36}$ and our recently developed carbon GAP. ${ }^{34}$ This po- tential has been validated in various ways, including by comparison to experimental PDFs for disordered carbon materials ${ }^{33}$ and by explicit simulation of deposition and growth of tetrahedral amorphous carbon ( $t a-C)$ films, reproducing the experi-

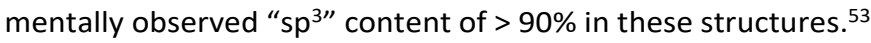
Each simulation started with 216 atoms per cell at mass densities between 1.5 and $2.5 \mathrm{~g} \mathrm{~cm}^{-3}$, using the protocol from ref. 34 . All MD simulations were done using quippy (which, together with the GAP code, is available at www.libatoms.org) with a Langevin thermostat and a timestep of $1 \mathrm{fs}$.

The $a-C$ precursors were gradually heated to $4,000 \mathrm{~K}$ over $100 \mathrm{ps}$ and held at that temperature for $400 \mathrm{ps}$. Compared with our previous work, ${ }^{33}$ we here annealed for longer time and at higher temperature, which induces a higher degree of ordering. ${ }^{50}$ After annealing at $4000 \mathrm{~K}$, the systems were cooled to 300 $\mathrm{K}$, undercoordinated atoms and long carbon chains pruned from the cells, as suggested before, ${ }^{33}$ followed by annealing at 3,000 $\mathrm{K}$ (100 ps), cooling/pruning, and another $3,000 \mathrm{~K}$ annealing run. Finally, cell volumes were optimised, and relaxations performed at the so-determined optimum volumes. Due to the removal of undercoordinated atoms and the volume optimisation, the final mass density and atom count is specific to each cell.

Our study covers a wide parameter space of structures and densities (Fig. 1a). We use the shortest-path ring statistics as a measure for structural order: ${ }^{54,55}$ in ideal graphite, all atoms are in six-membered (" $6 \mathrm{~m}$ ") rings, whereas odd-membered rings induce curvature (e.g., the $\mathrm{C}_{60}$ fullerene contains 20 six-membered and 12 five-membered rings $\left.{ }^{56}\right)$. The presence of odd rings in disordered carbons has been observed in $\mathrm{TEM}^{57,58}$ and also suggested based on pair distribution function (PDF) analyses. ${ }^{59}$ 
The structures from our simulations include one (labelled $\mathbf{1}$ ) that has no 5-/7-membered rings at all, but is much less dense than graphite, showing substantial bending of the sheets (ESI; Fig. S1). We emphasise that the cubic cell shape is kept fixed in our simulations; we confirmed that upon full (DFT) relaxation of $\mathbf{1}$, one obtains a structure more similar to graphite, but this is not the target here. We select sets of structures that let us study trends in either ordering (along the line $\mathbf{1} \rightarrow \mathbf{4}$ in Fig. 1a) or density $(\mathbf{6} \rightarrow \mathbf{8})$, while keeping the respective other property fixed. We also include one structure that is relatively similar to ideal graphite (5) but contains an extended defect (representing a "nanopore"; see below).

\section{Voltage-filling relations for disordered structures}

The experimentally relevant quantity for our study is the cell voltage as a function of filling (the latter being linked to the specific capacity). Generally, the voltage $U$ is obtained $a^{25}$

$$
U=-\frac{\Delta G}{n F}=-\frac{\left[G\left(M_{x} C_{y}\right)-x G(M)-y G(C)\right]}{n F}
$$

where $G$ is the Gibbs energy for a given component, $n$ denotes the (molar) number of electrons transferred, and $F$ is the Faraday constant. In computational practice, the above expression is normally approximated as

$$
U=-\frac{1}{x}\left[E_{\mathrm{DFT}}\left(M_{x} C_{y}\right)-x E_{\mathrm{DFT}}(M)-y E_{\mathrm{DFT}}(C)\right]
$$

where all total energies $E$ are obtained from DFT computations at the same level. Note that this approach is formally based on energies at zero Kelvin, and does therefore not take temperature into account; the validity of this approximation has been shown before. ${ }^{11}$ In this work, there are no crystallographically well-defined staging compounds with integer $x$ and $y$ (such as in ordered $\mathrm{LiC}_{6}$ ); instead, we have to sample a sufficiently large ensemble of candidate adsorption sites (Fig. 1b). We start with 20 independent computations at small $x$ and reduce the number of sampled cells to 10 and then to 5 at larger $x$, as the scatter between points decreases (Fig. 1C). The idea of exploring possible structures in a stochastic way, with only basic (physically motivated, such as hard-sphere) constraints is reminiscent of the $A b$ Initio Random Structure Searching (AIRSS) technique ${ }^{60,61}$ which has been widely used for battery materials. ${ }^{62-64}$

\section{Computational details}

DFT computations were performed using the projector augmented-wave method ${ }^{65}$ as implemented in VASP 5.4.4. ${ }^{66,67}$ Exchange and correlation were described using the optB88-vdW functional, ${ }^{68-71}$ which takes van der Waals ( $\mathrm{vdW}$ ) interactions into account. This level of theory was recently used to fit a GAP for pristine graphene, ${ }^{72}$ and computational parameters as in that work were used as appropriate. We confirmed that the chosen DFT method and settings accurately describe the lattice parameters of the experimentally known crystalline $M @ C$ phases (see ESI for details).
Initially, the atomic coordinates in all candidate structures were relaxed using DFT until residual forces fell below $0.01 \mathrm{eV}$ $\AA^{-1}$. In these computations, reciprocal space was sampled at the $\Gamma$ point, and an electronic smearing of $\sigma=0.2 \mathrm{eV}$ and a cut-off energy of $250 \mathrm{eV}$ were used; cell shape and volume were kept fixed (see above). Then, for the optimised structures, more accurate single-point computations were performed, employing a grid of $3 \times 3 \times 3 k$-points, $\sigma=0.1 \mathrm{eV}$, and a cut-off energy of 500 $\mathrm{eV}$. Convergence tests confirmed that increasing the grid to $5 \times$ $5 \times 5 k$-points changed the computed voltage values by no more than $0.01 \mathrm{~V}$ (ESI).

Projected electronic densities of states (DOS) were obtained from the self-consistent wavefunctions by analytic projection into a local auxiliary basis set of valence $s$ and $p$ orbitals, using the LOBSTER software (www.cohp.de), ${ }^{73,74}$ which has recently been applied to carbon materials for battery applications. ${ }^{33,75}$ Charges on atoms were computed using the Löwdin scheme ${ }^{76}$ as implemented in LOBSTER. ${ }^{77}$

\section{Results and discussion}

\section{Intercalation in disordered carbon structures}

With the optimised carbon structures 1-8 in hand (Fig. 1a), we proceed to simulate the insertion of metal atoms. DFT allows us to investigate arbitrary guest species directly-in this case, it is straightforward to add $\mathrm{Li}, \mathrm{Na}$, and $\mathrm{K}$ atoms in parallel runs. Due to the disordered nature of the structures, we need to sample several randomly chosen configurations; an example is given in Fig. 1c. In the latter figure, we also include experimental data for comparison: these depend strongly on the nature (e.g., the synthesis pathway) of the specific carbon material, and therefore the interpretation and comparison to DFT can be semiquantitative at best. That being said, we argue that the comparison of different model systems and the computed voltage-filling relations allows for useful insight that would be inaccessible without the large diversity of carbon structures we are able to sample here.

Table 1 DFT-computed adsorption energies for isolated $M$ atoms on ideal or defective graphene sheets, placing the atom on characteristic adsorption sites, as sketched. Negative values indicate favourable adsorption.

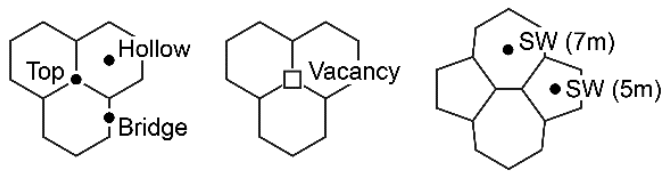

\begin{tabular}{llccc}
\hline & & \multicolumn{3}{c}{$\Delta E_{\text {ads }}(\mathrm{eV})$} \\
\cline { 3 - 5 } & & $M=\mathrm{Li}$ & $M=\mathrm{Na}$ & $M=\mathrm{K}$ \\
\hline \multirow{3}{*}{ Site } & -1.18 & -0.86 & -1.26 \\
& Top & -1.19 & -0.86 & -1.26 \\
& Bridge & -1.41 & -0.90 & -1.31 \\
\cline { 2 - 5 } & Hollow & -3.01 & -2.13 & -2.37 \\
& Vacancy & -1.82 & -1.15 & -1.68 \\
SW (5m ring) & -1.86 & -1.28 & -1.74 \\
\hline
\end{tabular}



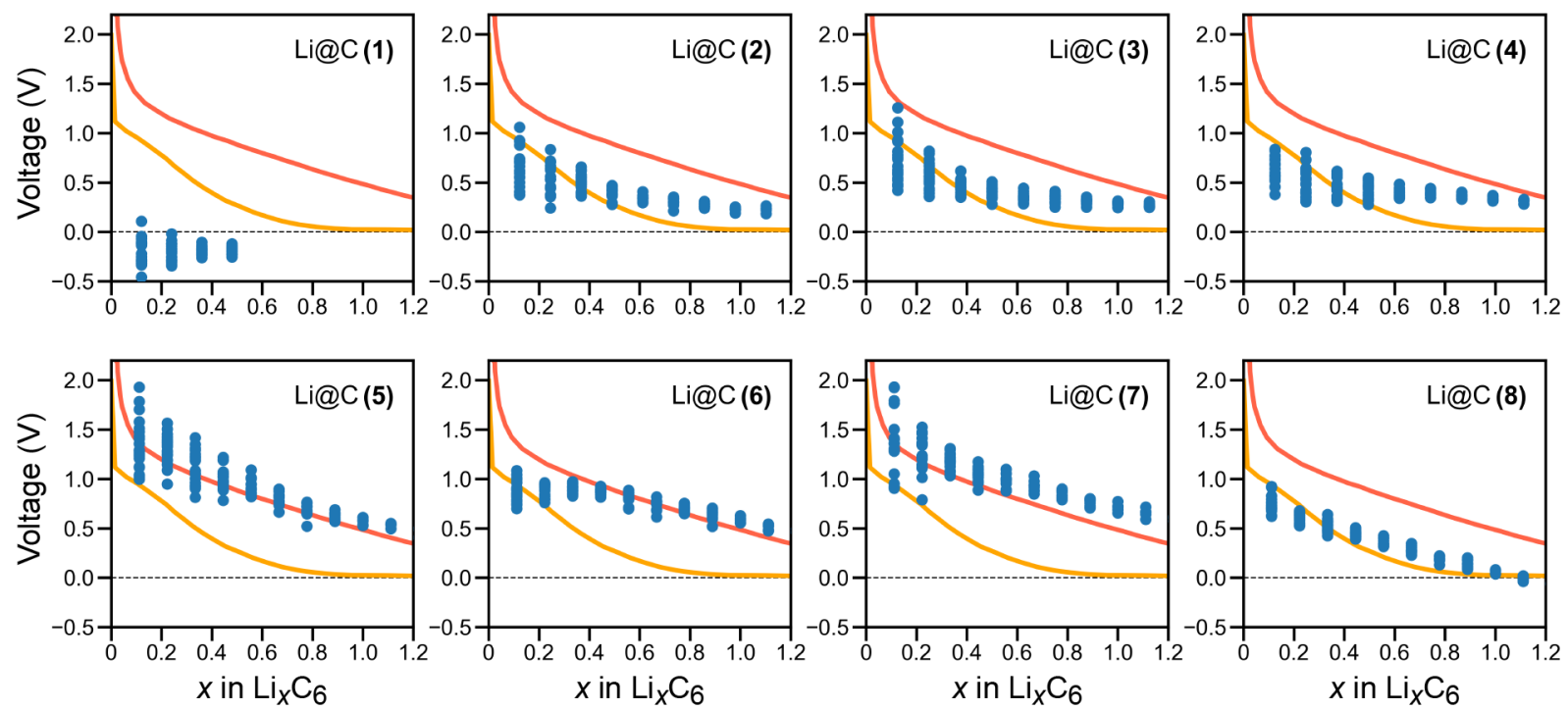

Fig. 2 Voltage-filling plots for carbon structures 1-8 in which Li atoms have been gradually inserted (as sketched in Fig. 1b and described in the Methodology section). Experimental data are taken from Legrain et al. (ref. 24; red) and Stevens and Dahn (using the "hard carbon" data from ref. 46; orange). DFT-computed values are shown as blue points.

To provide a point of reference, we begin by discussing metal adsorption energies in a simpler system: namely, on selected high-symmetry sites of pristine and defective graphene (Table 1). Such studies have been done before, ${ }^{47,78-80}$ but they are here repeated using the same DFT framework that we will use for the disordered structures, for full consistency. On a pristine graphene sheet, adsorption on the "hollow" site is always preferred over the "top" and "bridge" sites; this preference is much more strongly pronounced for Li than for the larger $\mathrm{Na}$ and $\mathrm{K}$ atoms. Among the three metals, the absolute adsorption energy (i.e., the stabilisation of an adsorbed atom) is lowest for $\mathrm{Na}$, in accord with the poor intercalation propensity of $\mathrm{Na}$ in graphite. ${ }^{6}$ Adsorption on a Stone-Wales (SW) defect and especially on a vacancy site is visibly more favourable than that on pristine graphene; again, the absolute stabilisation is largest for $\mathrm{Li}$ and smallest for $\mathrm{Na}$. Hence, structural defects (where they occur) will play a role early on in the adsorption process.

We now return to the central subject of this work, our disordered structures $\mathbf{1 - 8}$, focusing on Li intercalation for the moment (Fig. 2). The DFT results can be classified according to the properties of the underlying carbon framework:

(i) $\mathbf{1}$ is a structure made of sheets with ideal graphite-like topology (6m rings throughout), but with substantial non-planarity. We find that bending of carbon sheets alone, which 1 represents, is not a necessary or sufficient criterion for intercalation: almost all computed data points are at negative voltage (i.e., unfavourable insertion), and so the scan was terminated after a few steps.

(ii) 2-4 vary in their count of odd-membered rings, but they all have a similar initial density of $1.3-1.4 \mathrm{~g} \mathrm{~cm}^{-3}$, typical of porous carbon materials. The initial samples $(x<0.2)$ show a pronounced variation in maximum voltages, but once the structures approach larger filling $(x>0.5)$-that is, once the structural defects have been saturated-, their computed voltage-filling relations are quite similar, falling in between the two experimental datasets ${ }^{24,46}$ (red and orange lines in Fig. 2) in all three cases.

(iii) $\mathbf{5}$ is a structure with an open pore. Initially, the Li atoms readily intercalate, indicated by highly positive voltages (vs. $\mathrm{Li}^{+} / \mathrm{Li}$; favourable adsorption). Overall, the datapoints are in reasonable agreement with the dataset by Legrain et al. obtained for intercalation into amorphous carbon, ${ }^{24}$ although a truly quantitative comparison will require system sizes of much more than 200-250 atoms and the assessment of different pore shapes and sizes.

(iv) 6-8 are structures with varying density. They seem to have a maximum in voltages at intermediate density, at a value slightly lower than that of graphite (7; cf. Fig. 1a), whereas at too high density of the carbon framework (8) intercalation becomes distinctly less favourable.

Our simulation cells sample a wide range of structural motifs, such as odd-membered rings and open pores, which are expected to facilitate the adsorption and clustering of alkali metal atoms. The idealised computations in Table 1, in accord with previous literature, ${ }^{23,47}$ confirm that structural defects are preferred adsorption sites: these sites provide additional capacity and would result in high voltage (vs. $\mathrm{Li}^{+} / \mathrm{Li}$ ) when using carbon as anode. The DFT results for $\mathbf{2}-\mathbf{4}$ suggest that filling of such defects at the initial stage $(x<0.2)$ could have a significant effect on the voltage. We emphasise that these computations sample the (optimised) insertion structures at a given $x$, but not the dynamics of the entire gradual process: for example, defect sites on which Li is strongly bound can "trap" Li atoms, leading to an irreversible loss of capacity. To fully understand the reversibility, large-scale MD simulations of the charging / discharging and diffusion mechanisms are required. ML potentials could be suitable tools for such tasks, ${ }^{81}$ but the development and validation of these far exceeds the scope of the present paper. 

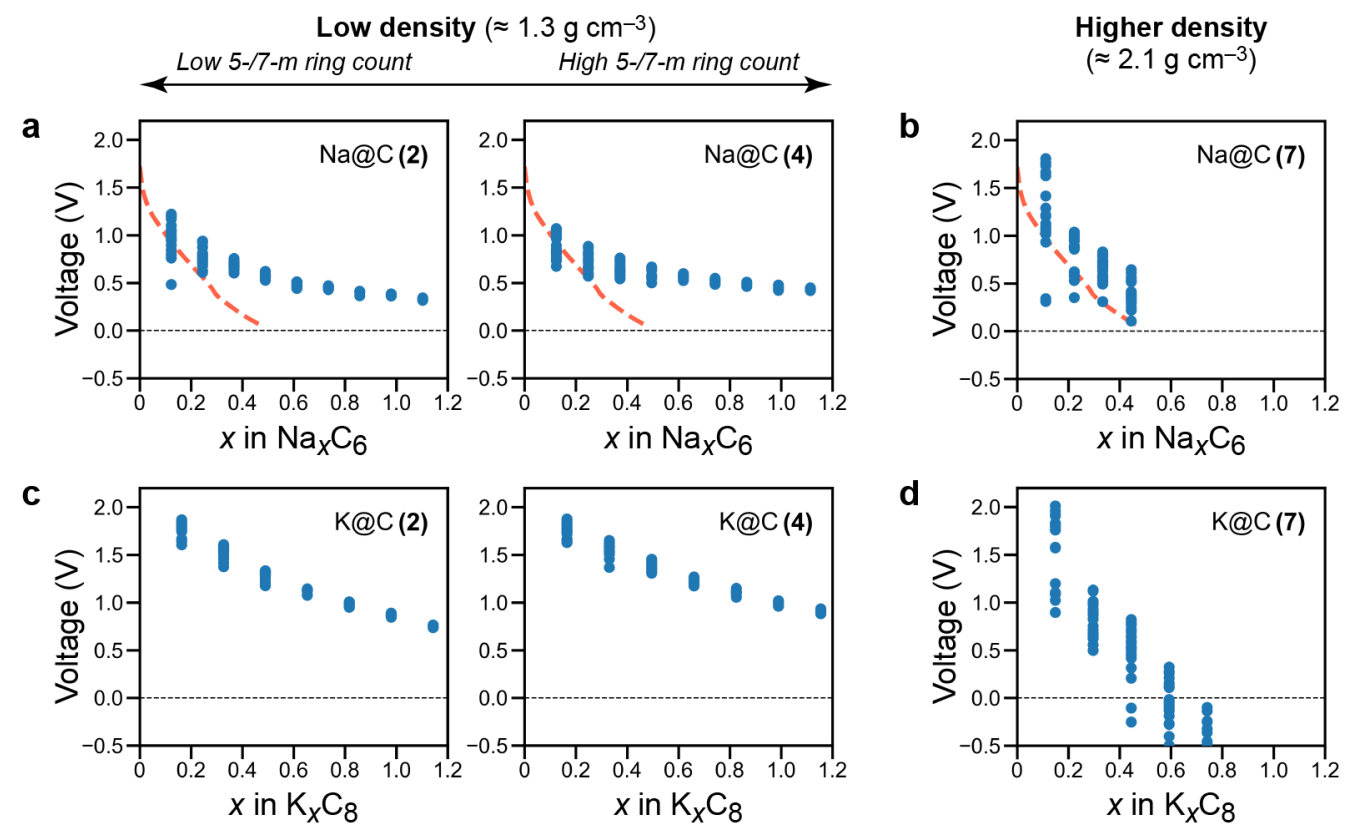

Fig. 3 Voltage-filling plots as in Fig. 2 but now for the intercalation of (a,b) Na and (c,d) K into structures 2, 4, and 7, respectively. Experimental data for Na intercalation (red dashed line) are taken from Legrain et al. (ref. 24); we do not know of reports on the reversible insertion of $\mathrm{K}$ in comparable amorphous carbon materials.

Moving in the vertical direction in Fig. 1a and comparing structures with a similar number of $5 \mathrm{~m}$ and $7 \mathrm{~m}$ rings (viz., 4, 6, $\mathbf{7}, \mathbf{8}$ ), but different density, clearly emphasises the role of the latter for intercalation. Low-density carbons exhibit a large space between carbon layers, making the adsorption of ions more similar to that on ideal graphene or, as recently observed, in bilayer graphene. ${ }^{82}$ On the contrary, for dense forms, distances between the layers are smaller and intercalated ions will begin to experience repulsion, causing the DFT voltage points to drop below zero for $\mathbf{8}$. Our data also suggest that the latter structure is better comparable to the more ordered material characterised by Stevens and Dahn (orange in Fig. 2), ${ }^{46}$ rather than to the highly disordered (ball-milled) amorphous samples of Legrain et al. (red in Fig. 2). Summarising thus far, the density appears to be an important factor to be considered when designing disordered carbon materials for energy storage.

Having studied Li intercalation in some detail, we now look at the heavier homologues, $\mathrm{Na}$ and $\mathrm{K}$. Exploratory computations showed that not all eight model carbon systems are relevant in this case: intercalation in $\mathbf{1}$ is not favoured, similar to the case of Li (Fig. 2a), and the dense structure 8 does not readily accommodate the larger ions (see Fig. S2 in the ESI). We therefore focus on selected structural models at densities commensurate with different carbonaceous materials. The results are collected in Fig. 3.

At low densities, the predicted voltage-filling relations are reminiscent of those for $\mathrm{Li}$; introducing structural disorder in the form of $5 \mathrm{~m} / 7 \mathrm{~m}$ rings (which is small in 2 but large in 4 ) in our models has a very limited effect on the overall shape of the computed voltage profile. For $\mathrm{Na}$ intercalation (Fig. 3a), both 2 and 4 lead to a substantially higher voltage and capacity than the experiment to which we compare, from $x \approx 0.2$ onwards.
They are therefore not suitable proxies for modelling this specific material: indeed, super $P$ carbon materials $\left(\approx 1.6 \mathrm{~g} \mathrm{~cm}^{-3}\right)$ are more disordered and slightly denser than the simulated models. There is a discernible variation in $\mathrm{Na}$ intercalation at small filling (i.e., the voltage ranges differ in $\mathbf{2}$ and $\mathbf{4}$ for $x<0.2$ ); no such effect is seen for $\mathrm{K}$ (Fig. 3c). The predicted voltages, in all these low-density cases, remain positive throughout the range of $x$ values surveyed, exceeding the (hypothetical) limit of " $\mathrm{NaC}_{6}$ " ( $x=1.0$ in Fig. 3a) and the experimentally known composition $\mathrm{KC}_{8}(x=1.0$ in Fig. $3 \mathrm{~b})$. The presence of voids, therefore, is expected to principally enhance the uptake of the heavier alkali atoms.

For structure 7 , which has a higher density $\left(\approx 2.1 \mathrm{~g} \mathrm{~cm}^{-3}\right)$ approaching that of graphite, the slope of the Na voltage-filling data agrees better with experiment (Fig. 3b). We also explored $\mathrm{K}$ intercalation in $\mathbf{7}$, where the voltages reach zero (indicating unfavourable intercalation) at a $\mathrm{K}$ content of around half that of the ideal crystalline compound $\mathrm{KC}_{8}$ (Fig. $3 \mathrm{~d}$ ). For future experiments on $\mathrm{K}$-ion battery anodes even more so than for $\mathrm{Na}$, lowerdensity carbons may be of interest.

Concluding the discussion of computed voltage-filling relations, it should be noted that a solid electrolyte interphase (SEI) will form between the carbon anode and an organic electrolyte during the first charge/discharge cycle. ${ }^{8,83,84}$ As a consequence of SEI formation, the alkali ions now need to pass through the layer to intercalate in the carbon materials; the precise nature of this process is expected to differ between $\mathrm{Li}, \mathrm{Na}$, and $\mathrm{K} .{ }^{8,83,84}$ The SEl layer could be considered as a resistor and its presence would slightly lower the voltage curve compared to our prediction. Solid-state NMR could be used to probe the SEI, ${ }^{85}$ and elucidating the mechanisms of how ions pass through the layer could benefit from further (DFT- or ML-driven) MD studies. 
a

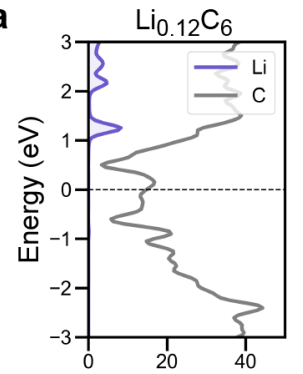

b
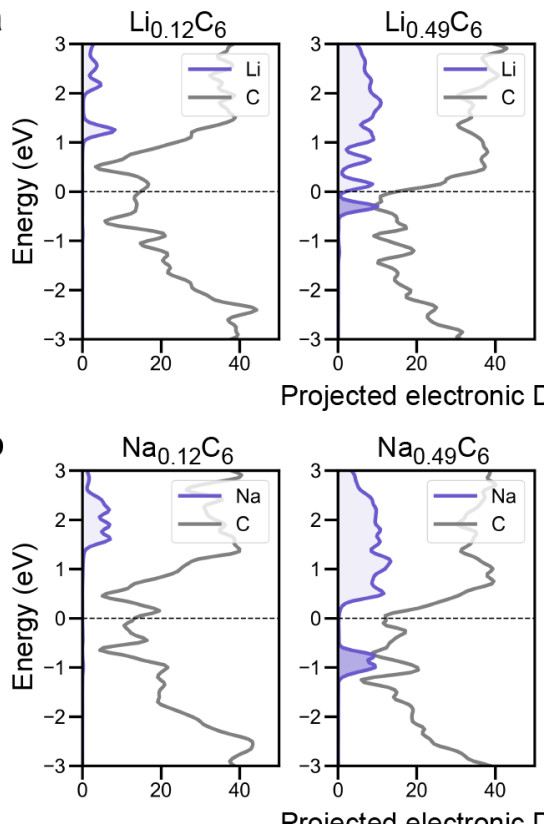

Projected electronic DOS
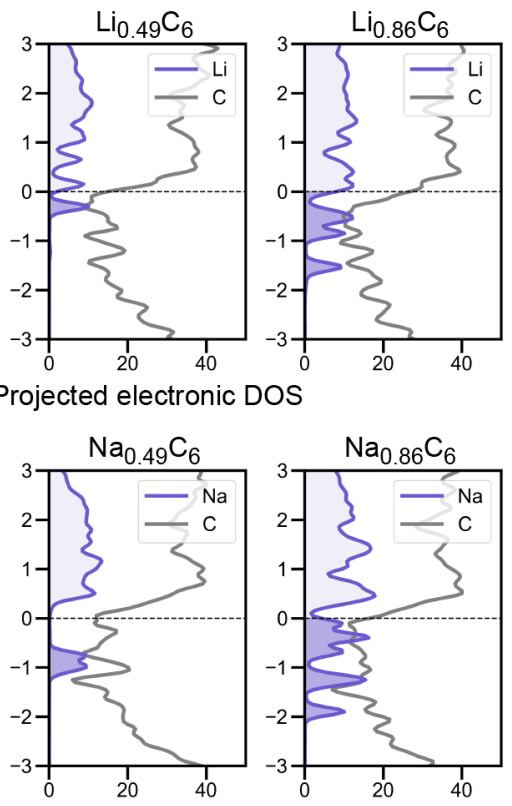

Projected electronic DOS

C
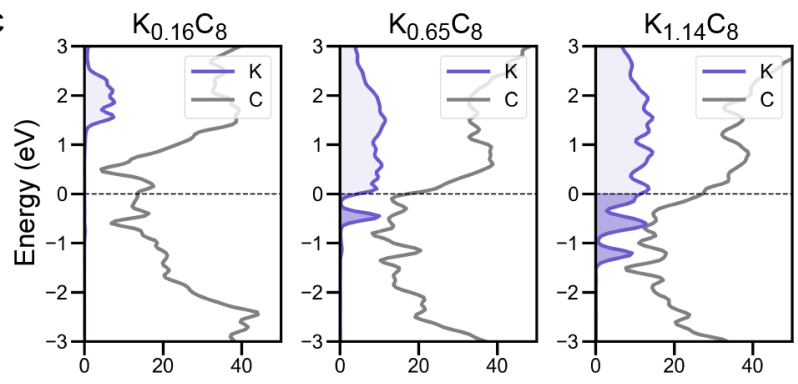

Projected electronic DOS

Fig. 4 Electronic densities of states (DOS), computed using DFT and atomically resolved by projection into a local basis (Methods), providing a fingerprint of electronic structure at various degrees of filling. Projections onto the $\mathrm{C}$ atoms (grey) and metal atoms (blue) are shown. The Fermi level is set as energy zero and indicated by a dashed line.

\section{Electronic structure}

With the optimised atomic structures available, electronicstructure computations were carried out to better understand the intercalation properties. We compare, in Fig. 4, atomically resolved electronic densities of states (DOS) for the three alkali metals, showing three selected datasets for each, from low to high filling. Due to the presence of defects and disorder, carbon systems from such simulations exhibit a finite DOS at the Fermi level. ${ }^{33}$ Initially (left-hand side), the DOS contributions by the metal atoms are all above the Fermi level (dashed line); this corresponds to an unfilled $s$ valence orbital and therefore to an ionic state in all three cases $\left(\mathrm{Li}^{+}, \mathrm{Na}^{+}, \mathrm{K}^{+}\right)$. With increasing filling, larger and larger contributions below the Fermi level are seen in the projected DOS for the metal atoms (middle and righthand-side panels in Fig. $4 \mathrm{a}-\mathrm{c}$ ). Such mechanisms have been previously discussed for Li on graphene ${ }^{86}$ and $\mathrm{Na}$ in a "porous" carbon model system. ${ }^{33}$ Our present computations, starting from the same carbon frameworks for $\mathrm{Li}, \mathrm{Na}$, and $\mathrm{K}$ (and therefore ensuring direct comparability), suggest the cross-over from ionic to metallic nature to be a universal feature of all three alkali-metal species being intercalated into carbon materials. a

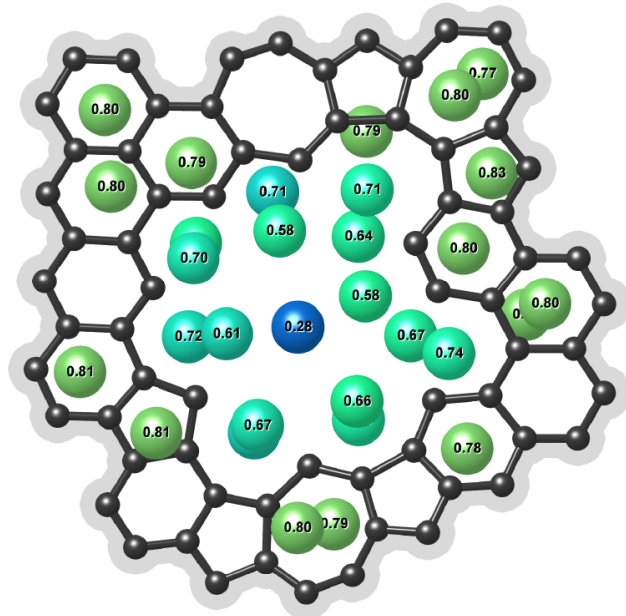

b

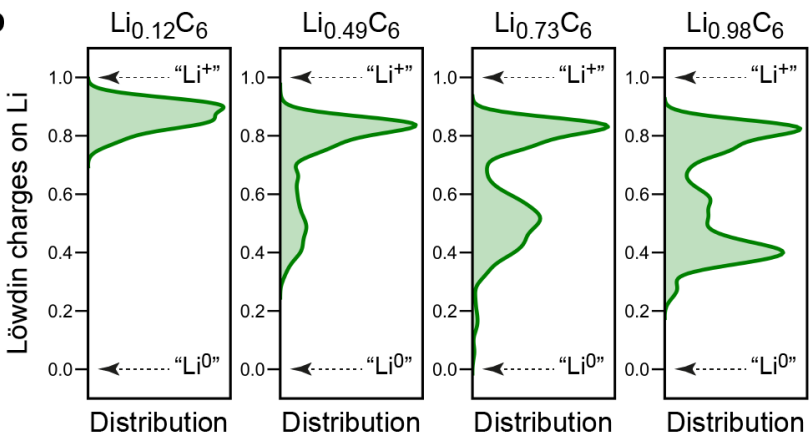

Fig. 5 Atomic charges on metal atoms in disordered carbons, as dependent on the loca structure and the degree of filling. (a) Example snapshot from Li intercalation in 5, in which the Li atoms are drawn as large spheres and labelled with the individual Löwdin charges (as computed using LOBSTER; see Methodology section). Carbon atoms are drawn as smaller black spheres, and shading indicates that the $2 \mathrm{D}$-like sheet continues outside the region that is shown, whereas there is a large defect ("nanopore") in the centre. There is a clear distinction between atoms adsorbed on top of rings on the one hand, and clustered atoms in the centre of the pore on the other hand. In the outer region, the preferential adsorption of atoms on hollow sites (rather than directly atom atoms or bond) is qualitatively consistent with the results for ideal graphene in Table 1. (b) Charges for Li atom intercalation with increasing filling, drawn as kernel density estimates ("smoothed histograms") for four representative choices of $x$ in $\mathrm{Li}_{x} \mathrm{C}_{6}$.

\section{Charges on atoms: ionic versus metallic nature}

It has been deduced from operando NMR experiments that charge is gradually transferred during $\mathrm{Na}$ insertion in disordered carbons, starting with $\mathrm{Na}^{+}$ions and then moving towards $\mathrm{Na}^{0} .87$ To supplement these findings with first-principles simulations, we compute charges on atoms; this largely extends our earlier proof-of-concept, where we studied a few instances of $\mathrm{Na}$ intercalation. ${ }^{33}$ An example, now for the case of $\mathrm{Li}$, is given in Fig. $5 \mathrm{a}$ : atoms adsorbed near the edge of the pore mainly reside on the "hollow" sites of $6 \mathrm{~m}$ and odd-membered rings (consistent with the data for idealised graphene in Table 1), and their charges are on the order of 0.8 , close to ideal $\mathrm{Li}^{+}$. The majority of atoms is found near the edge of the nanopore, in which case the charges are somewhat smaller but still clearly supporting a partially ionic nature of Li. In the centre of the pore, finally, we observe one $\mathrm{Li}$ atom that is surrounded by many others, but that is not close to any $\mathrm{C}$ atom of the host framework. In this case, the computed charge is 0.28 , bringing the electronic nature of this atom very close to the metallic state. 


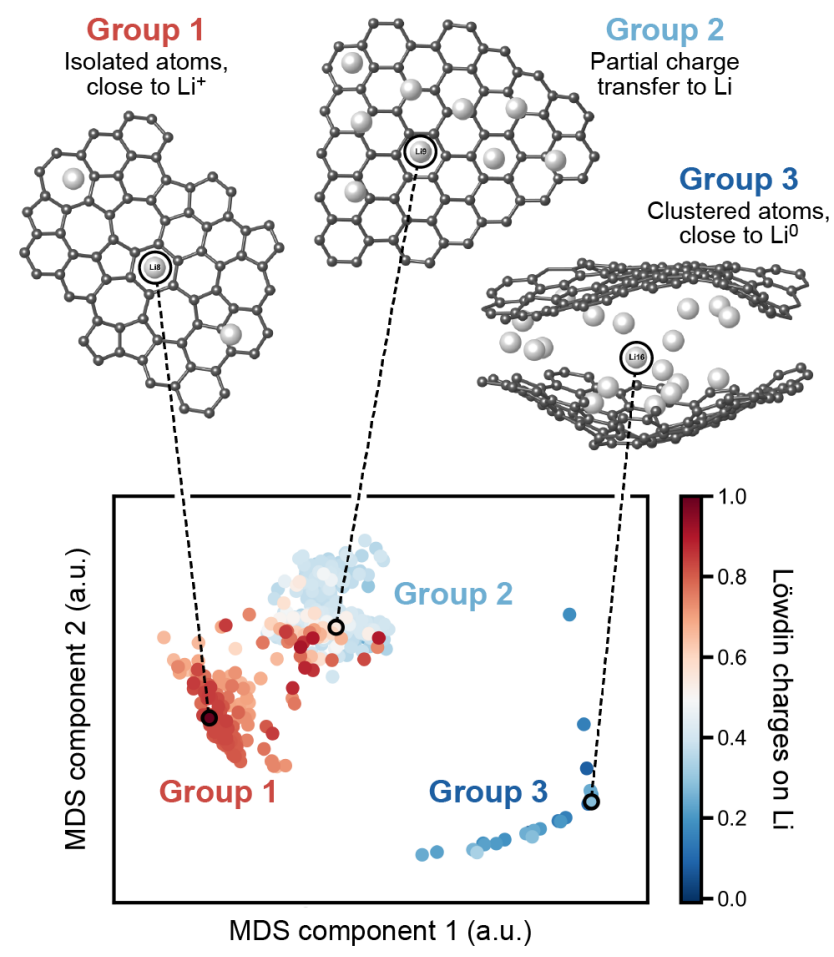

Fig. 6 Unsupervised machine learning of Li-atom environments in a range of intercalated structures. A structural similarity map (obtained by multidimensional scaling, MDS) based on SOAP distances between environments is drawn such that the most dissimilar points are furthest away, and vice versa. Colour coding indicates the computed charges for each given atom. Structural drawings are provided for three representative environments (circled), corresponding to high, medium, and low Löwdin charges on Li atoms.

A more quantitative view is enabled by collecting charge values over several atomic environments, at various degrees of filling ( $x$ in $\mathrm{Li}_{x} \mathrm{C}_{6}$ ) and comparing these results side-by-side (Fig. $5 \mathrm{~b}$ ). In accord with the PDOS data that we have discussed above, the charge distributions gradually shift down and lead to an apparently bimodal distribution, with one peak near 0.8 (persisting even at very high filling, close to $\mathrm{LiC}_{6}$ ), and one peak building up between Löwdin charges of 0.6 and 0.3 . This splitting suggests that there are distinctly different groups of $\mathrm{Li}$ atoms in carbon anode materials. However, this distribution alone does not yet reveal the structural and chemical origins of these groups.

We therefore use the Smooth Overlap of Atomic Positions (SOAP) kernel, ${ }^{88}$ a structural similarity measure initially used for GAP fitting but having more general implications, to analyse local structures. SOAP quantifies the similarity of two atomic environments $i$ and $j$ on a scale from $k_{i j}=0$ (fully dissimilar) to $k_{i j}=1$ (identical up to symmetry operations and within a specified cut-off radius), where the SOAP kernel $k_{i j}$ is defined in ref. 88; parameters are taken from ref. 81. Visualising the so-obtained (dis)similarity between atomic environments can yield useful information. ${ }^{89}$ For example, a technique called clustering can be employed to identify the most relevant chemical sites in amorphous structures, as shown by Caro et al. for carbon materials. ${ }^{41}$ These approaches are examples for the wider area of "unsupervised" machine learning, in which one aims to extract information from un-labelled datasets; the usefulness of such techniques for chemistry has been highlighted very recently. ${ }^{90}$
In Fig. 6, we visualise the structural distances and thus the relationships between different environments of $L i$ atoms in DFT-optimised structures at various degrees of filling. We compute the distance $d$ of sites from one another, defined as

$$
d(i, j)=\sqrt{2-2 k_{i j}}
$$

and use a dimensionality reduction technique to draw a 2D map that represents these distances. We then colour-code the data points by (DFT-computed) charges on the respective atoms, aiming to discover not only structural but structure-property relationships.

Interestingly, the Li environments appear to fall not into two groups (as the apparently bimodal distributions in Fig. 5b might have suggested), but into three groups, based on the local environments involved. We observe (1) isolated atoms, which the colour-coding in Fig. 6 confirms to have an almost fully positive charge, (2) clustered atoms with approximately half a unit charge, with a gradual transition between the two, and then, structurally far away, (3) metallic-like atoms with charges close to zero. It is expected that such analysis techniques will be useful in the future when studying disordered carbons where traditionally used labels such as the "hollow", "top", and "bridge" adsorption sites (Table 1) cannot be easily applied due to the structural complexity and vast amounts of data; instead, kernelbased distance maps can be generated in an automated fashion and without the need for pre-defining any structural motifs. We stress that they are not restricted to carbon but could prove useful for other disordered battery materials as well.

\section{Conclusions and outlook}

Machine learning and DFT methods have been combined to study the intercalation of alkali metal atoms in model carbon systems, comparing all three species $(\mathrm{Li}, \mathrm{Na}, \mathrm{K})$ that are relevant to batteries. Performing up to 20 independent computations for each system and step of filling makes it possible (and is, in fact, required) to gain stochastic insight into the atomic-scale processes, charges on atoms, and the mechanisms by which metal ions are inserted. These mechanisms are reflected in predicted voltage-filling relations (providing a direct link to experiments within the limits of finite model system sizes). Having access to representative structures also makes it straightforward to compute and analyse electronic-structure fingerprints (such as projected densities-of-states and atomic charges), which we have here presented for all three metals side-by-side.

It is hoped that the simulation protocols and structural data provided in this work can directly enable more detailed DFT studies in the future-selecting, from the wide parameter space of disorder and densities, the most representative model system to represent a given problem (such as a disordered region in "hard" carbons). The strategy outlined here could thus become a useful general approach to understand, and ultimately, to optimise, disordered carbon materials for future battery technologies. 


\section{Conflicts of interest}

There are no conflicts to declare.

\section{Acknowledgements}

We thank Professor Clare P. Grey for useful discussions. J.-X.H. acknowledges support from the Graduate Overseas Exchange Program of Xiamen University and the Collaborative Innovation Center of Chemistry for Energy Materials (iChEM) for a research visit at the University of Cambridge, during which he performed the research reported herein. G.C. acknowledges EPSRC grant EP/P022596/1. J.C. acknowledges National Natural Science Foundation of China (Grant Nos. 2181101075, 91745103 and 21621091). V.L.D. acknowledges a Leverhulme Early Career Fellowship and support from the Isaac Newton Trust.

\section{Data access statement}

Data supporting this publication are provided as Electronic Supplementary Information (ESI); further data will be made available through an online repository.

\section{Notes and references}

Y. P. Wu, E. Rahm and R. Holze, Carbon anode materials for lithium ion batteries, J. Power Sources, 2003, 114, 228-236.

2 N. A. Kaskhedikar and J. Maier, Lithium storage in carbon nanostructures, Adv. Mater., 2009, 21, 2664-2680. S.-W. Kim, D.-H. Seo, X. Ma, G. Ceder and K. Kang, Electrode Materials for Rechargeable Sodium-Ion Batteries: Potential Alternatives to Current Lithium-Ion Batteries, Adv. Energy Mater., 2012, 2, 710-721. N. Nitta, F. Wu, J. T. Lee and G. Yushin, Li-ion battery materials: present and future, Mater. Today, 2015, 18, 252-264. E. Irisarri, A. Ponrouch and M. R. Palacin, Review-Hard Carbon Negative Electrode Materials for Sodium-Ion Batteries, J. Electrochem. Soc., 2015, 162, A2476-A2482. Y. Liu, B. V Merinov and W. A. Goddard, Origin of low sodium capacity in graphite and generally weak substrate binding of $\mathrm{Na}$ and $\mathrm{Mg}$ among alkali and alkaline earth metals, Proc. Natl. Acad. Sci., U. S. A., 2016, 113, 3735-3739.

S. Komaba, T. Hasegawa, M. Dahbi and K. Kubota, Potassium intercalation into graphite to realize high-voltage / high-power potassium-ion batteries and potassium-ion capacitors, Electrochem. Commun., 2015, 60, 172-175. Z. Jian, W. Luo and X. Ji, Carbon Electrodes for K-Ion Batteries, J. Am. Chem. Soc., 2015, 137, 11566-11569.

9 J. Zhao, X. Zou, Y. Zhu, Y. Xu and C. Wang, Electrochemical Intercalation of Potassium into Graphite, Adv. Funct. Mater., 2016, 26, 8103-8110.

10 M. S. Islam and C. A. J. Fisher, Lithium and sodium battery cathode materials: computational insights into voltage, diffusion and nanostructural properties, Chem. Soc. Rev., 2014, 43, 185-204.

11 A. Urban, D. Seo and G. Ceder, Computational understanding of $\mathrm{Li}$ ion batteries, npj Comput. Mater., 2016, 2, 16002.

13

energy materials using density functional theory, Nat. Rev. Mater., 2016, 1, 1-13.

K. R. Kganyago and P. E. Ngoepe, Structural and electronic properties of lithium intercalated graphite $\mathrm{LiC}_{6}$, Phys. Rev. B, 2003, 68, 205111.

K. Toyoura, Y. Koyama, A. Kuwabara, F. Oba and I. Tanaka, Firstprinciples approach to chemical diffusion of lithium atoms in a graphite intercalation compound, Phys. Rev. B, 2008, 78, 214303. K. Persson, Y. Hinuma, Y. S. Meng, A. Van Der Ven and G. Ceder, Thermodynamic and kinetic properties of the Li-graphite system from first-principles calculations, Phys. Rev. B, 2010, 82, 125416. V. Pande and V. Viswanathan, Robust high-fidelity DFT study of the lithium-graphite phase diagram, Phys. Rev. Mater., 2018, 2, 125401

E. Ziambaras, J. Kleis, E. Schröder and P. Hyldgaard, Potassium intercalation in graphite: A van der Waals density-functional study, Phys. Rev. B, 2007, 76, 155425.

W. Luo, J. Wan, B. Ozdemir, W. Bao, Y. Chen, J. Dai, H. Lin, Y. Xu, F. $\mathrm{Gu}, \mathrm{V}$. Barone and L. Hu, Potassium Ion Batteries with Graphitic Materials, Nano Lett., 2015, 15, 7671-7677.

Z. Xu, X. Lv, J. Chen, L. Jiang, Y. Lai and J. Li, Dispersion-corrected DFT investigation on defect chemistry and potassium migration in potassium-graphite intercalation compounds for potassium ion batteries anode materials, Carbon, 2016, 107, 885-894.

J. Liu, T. Yin, B. Tian, B. Zhang, C. Qian, Z. Wang, L. Zhang, P. Liang, Z. Chen, J. Yan, X. Fan, J. Lin, X. Chen, Y. Huang, K. P. Loh and Z. X. Shen, Unraveling the Potassium Storage Mechanism in Graphite Foam, Adv. Energy Mater., 2019, 1900579.

P. -c. Tsai, S.-C. Chung, S. -k. Lin and A. Yamada, Ab initio study of sodium intercalation into disordered carbon, J. Mater. Chem. A, 2015, 3, 9763-9768.

Z. Jian, C. Bommier, L. Luo, Z. Li, W. Wang, C. Wang, P. A. Greaney and X. Ji, Insights on the Mechanism of Na-lon Storage in Soft Carbon Anode, Chem. Mater., 2017, 29, 2314-2320.

C. Bommier, X. Ji and P. A. Greaney, Electrochemical Properties and Theoretical Capacity for Sodium Storage in Hard Carbon: Insights from First Principles Calculations, Chem. Mater., 2019, 31, 658-677.

F. Legrain, J. Sottmann, K. Kotsis, S. Gorantla, S. Sartori and S. Manzhos, Amorphous (Glassy) Carbon, a Promising Material for Sodium Ion Battery Anodes: a Combined First-Principles and Experimental Study, J. Phys. Chem. C, 2015, 119, 13496-13501. M. K. Y. Chan, C. Wolverton and P. Greeley, First Principles Simulations of the Electrochemical Lithiation and Delithiation of Faceted Crystalline Silicon, J. Am. Chem. Soc., 2012, 134, 1436214374.

R. C. Powles, N. A. Marks and D. W. M. Lau, Self-assembly of $s p^{2}$ bonded carbon nanostructures from amorphous precursors, Phys. Rev. B, 2009, 79, 075430.

J. C. Palmer, A. Llobet, S.-H. Yeon, J. E. Fischer, Y. Shi, Y. Gogotsi and K. E. Gubbins, Modeling the structural evolution of carbidederived carbons using quenched molecular dynamics, Carbon, 2010, 48, 1116-1123.

S. Schweizer, R. Meissner, M. Amkreutz, K. Thiel, P. Schiffels, J. Landwehr, B. J. M. Etzold and J.-R. Hill, Molecular Modeling of Microporous Structures of Carbide-Derived Carbon Based Supercapacitors, J. Phys. Chem. C, 2017, 121, 7221-7231. 
29 L. M. Mejía-Mendoza, M. Valdez-Gonzalez, J. Muñiz, U. Santiago, A. K. Cuentas-Gallegos and M. Robles, A theoretical approach to the nanoporous phase diagram of carbon, Carbon, 2017, 120, 233-243.

30 R. Ranganathan, S. Rokkam, T. Desai and P. Keblinski, Generation of amorphous carbon models using liquid quench method: A reactive molecular dynamics study, Carbon, 2017, 113, 87-99.

31 M. Raju, P. Ganesh, P. R. C. Kent and A. C. T. van Duin, Reactive Force Field Study of Li/C Systems for Electrical Energy Storage, J. Chem. Theory Comput., 2015, 11, 2156-2166.

E. Hjertenaes, A. Q. Nguyen and H. Koch, A ReaxFF force field for sodium intrusion in graphitic cathodes, Phys. Chem. Chem. Phys., 2016, 18, 31431-31440.

33 V. L. Deringer, C. Merlet, Y. Hu, T. H. Lee, J. A. Kattirtzi, O. Pecher, G. Csányi, S. R. Elliott and C. P. Grey, Towards an atomistic understanding of disordered carbon electrode materials, Chem. Commun., 2018, 54, 5988-5991.

V. L. Deringer and G. Csányi, Machine learning based interatomic potential for amorphous carbon, Phys. Rev. B, 2017, 95, 094203. J. Behler and M. Parrinello, Generalized neural-network representation of high-dimensional potential-energy surfaces., Phys. Rev. Lett., 2007, 98, 146401.

A. P. Bartók, M. C. Payne, R. Kondor and G. Csányi, Gaussian Approximation Potentials: The Accuracy of Quantum Mechanics, without the Electrons, Phys. Rev. Lett., 2010, 104, 136403. J. S. Smith, O. Isayev and A. E. Roitberg, ANI-1: an extensible neural network potential with DFT accuracy at force field computational cost, Chem. Sci., 2017, 8, 3192-3203.

S. Chmiela, A. Tkatchenko, H. E. Sauceda, I. Poltavsky, K. T. Schütt and K. Müller, Machine learning of accurate energy-conserving molecular force fields, Sci. Adv., 2017, 3, e1603015.

H. Wang, L. Zhang, J. Han and W. E, DeePMD-kit: A deep learning package for many-body potential energy representation and molecular dynamics, Comput. Phys. Commun., 2018, 228, 178184.

40 V. L. Deringer, M. A. Caro, R. Jana, A. Aarva, S. R. Elliott, T. Laurila, G. Csányi and L. Pastewka, Computational Surface Chemistry of Tetrahedral Amorphous Carbon by Combining Machine Learning and DFT, Chem. Mater., 2018, 30, 7438-7445

41 M. A. Caro, A. Aarva, V. L. Deringer, G. Csányi and T. Laurila, Reactivity of Amorphous Carbon Surfaces: Rationalizing the Role of Structural Motifs in Functionalization using Machine Learning, Chem. Mater., 2018, 30, 7446-7455.

42 N. Artrith, A. Urban and G. Ceder, Constructing first-principles phase diagrams of amorphous $\mathrm{Li}_{x} \mathrm{Si}$ using machine-learningassisted sampling with an evolutionary algorithm, J. Chem. Phys., 2018, 148, 241711.

43 B. Onat, E. D. Cubuk, B. D. Malone and E. Kaxiras, Implanted neural network potentials: Application to Li-Si alloys, Phys. Rev. B, 2018 97, 094106.

S. Shi, J. Gao, Y. Liu, Y. Zhao, Q. Wu, W. Ju, C. Ouyang and R. Xiao,

Multi-scale computation methods: Their applications in lithium-ion battery research and development, Chinese Phys. B, 2016, 25, 018212.

Y. Liu, T. Zhao, W. Ju and S. Shi, Materials discovery and design using machine learning, J. Materiomics, 2017, 3, 159-177.

Sodium Insertion in Carbon Materials, J. Electrochem. Soc., 2001, 148, A803.

X. Fan, W. T. Zheng and J.-L. Kuo, Adsorption and Diffusion of Li on Pristine and Defective Graphene, ACS Appl. Mater. Interfaces, 2012, 4, 2432-2438.

L.-J. Zhou, Z. F. Hou and L.-M. Wu, First-Principles Study of Lithium Adsorption and Diffusion on Graphene with Point Defects, J. Phys. Chem. C, 2012, 116, 21780-21787.

C. de Tomas, I. Suarez-Martinez and N. A. Marks, Graphitization of amorphous carbons: A comparative study of interatomic potentials, Carbon, 2016, 109, 681-693.

C. de Tomas, I. Suarez-Martinez, F. Vallejos-Burgos, M. J. López, K. Kaneko and N. A. Marks, Structural prediction of graphitization and porosity in carbide-derived carbons, Carbon, 2017, 119, 1-9. T. B. Shiell, D. G. McCulloch, D. R. McKenzie, M. R. Field, B. Haberl, R. Boehler, B. A. Cook, C. De Tomas, I. Suarez-Martinez, N. A. Marks and J. E. Bradby, Graphitization of Glassy Carbon after Compression at Room Temperature, Phys. Rev. Lett., 2018, 120, 215701.

N. A. Marks, Generalizing the environment-dependent interaction potential for carbon, Phys. Rev. B, 2000, 63, 035401.

M. A. Caro, V. L. Deringer, J. Koskinen, T. Laurila and G. Csányi, Growth Mechanism and Origin of High $s p^{3}$ Content in Tetrahedral Amorphous Carbon, Phys. Rev. Lett., 2018, 120, 166101.

D. S. Franzblau, Computation of ring statistics for network models of solids, Phys. Rev. B, 1991, 44, 4925-4930.

S. R. Elliott, Medium-range structural order in covalent amorphous solids, Nature, 1991, 354, 445-452.

H. W. Kroto, J. R. Heath, S. C. O’Brien, R. F. Curl and R. E. Smalley, $C_{60}$ : Buckminsterfullerene, Nature, 1985, 318, 162-163.

P. J. F. Harris, Z. Liu and K. Suenaga, Imaging the atomic structure of activated carbon, J. Phys. Condens. Matter, 2008, 20, 362201.

J. Kotakoski, A. V Krasheninnikov, U. Kaiser and J. C. Meyer, From Point Defects in Graphene to Two-Dimensional Amorphous Carbon, Phys. Rev. Lett., 2011, 106, 105505.

A. C. Forse, C. Merlet, P. K. Allan, E. K. Humphreys, J. M. Griffin, M. Aslan, M. Zeiger, V. Presser, Y. Gogotsi and C. P. Grey, New Insights into the Structure of Nanoporous Carbons from NMR, Raman, and Pair Distribution Function Analysis, Chem. Mater., 2015, 27, 6848-6857.

C. J. Pickard and R. J. Needs, High-pressure phases of silane, Phys. Rev. Lett., 2006, 97, 045504.

C. J. Pickard and R. J. Needs, Ab initio random structure searching, J. Phys. Condens. Matter, 2011, 23, 053201.

A. J. Morris, C. P. Grey and C. J. Pickard, Thermodynamically stable lithium silicides and germanides from density functional theory calculations, Phys. Rev. B, 2014, 90, 054111.

M. Mayo, K. J. Griffith, C. J. Pickard and A. J. Morris, Ab Initio Study of Phosphorus Anodes for Lithium- and Sodium-Ion Batteries, Chem. Mater., 2016, 28, 2011-2021.

E. Marbella, M. L. Evans, M. F. Groh, J. Nelson, K. J. Griffith, A. J. Morris and C. P. Grey, Sodiation and Desodiation via Helical Phosphorus Intermediates in High-Capacity Anodes for Sodium-Ion Batteries, J. Am. Chem. Soc., 2018, 140, 7994-8004.

P. E. Blöchl, Projector augmented-wave method, Phys. Rev. $B$ 1994, 50, 17953-17979.

G. Kresse and J. Furthmüller, Efficient Iterative Schemes for Ab 
Initio Total-Energy Calculations Using a Plane-Wave Basis Set, Phys. Rev. B, 1996, 54, 11169-11186.

67 G. Kresse and D. Joubert, From ultrasoft pseudopotentials to the projector augmented-wave method, Phys. Rev. B, 1999, 59, 17581775.

68 M. Dion, H. Rydberg, E. Schröder, D. C. Langreth and B. I. Lundqvist, Van der Waals Density Functional for General Geometries, Phys. Rev. Lett., 2004, 92, 246401.

69 J. Klimeš, D. R. Bowler and A. Michaelides, Chemical accuracy for the van der Waals density functional, J. Phys. Condens. MatterMatter, 2009, 22, 022201.

70 G. Román-Pérez and J. M. Soler, Efficient Implementation of a van der Waals Density Functional: Application to Double-Wall Carbon Nanotubes, Phys. Rev. Lett., 2009, 103, 096102.

71 J. Klimeš, D. R. Bowler and A. Michaelides, Van der Waals density functionals applied to solids, Phys. Rev. B, 2011, 83, 195131.

72 P. Rowe, G. Csányi, D. Alfè and A. Michaelides, Development of a machine learning potential for graphene, Phys. Rev. B, 2018, 97, 054303.

73 S. Maintz, V. L. Deringer, A. L. Tchougréeff and R. Dronskowski, Analytic projection from plane-wave and PAW wavefunctions and application to chemical-bonding analysis in solids, J. Comput. Chem., 2013, 34, 2557-2567.

74 S. Maintz, V. L. Deringer, A. L. Tchougréeff and R. Dronskowski, LOBSTER: A tool to extract chemical bonding from plane-wave based DFT, J. Comput. Chem., 2016, 37, 1030-1035.

75 K. Ni, X. Wang, Z. Tao, J. Yang, N. Shu, J. Ye, F. Pan, J. Xie, Z. Tan, X. Sun, J. Liu, Z. Qi, Y. Chen, X. Wu and Y. Zhu, In Operando Probing of Lithium-Ion Storage on Single-Layer Graphene, Adv. Mater., 2019, 31, 1808091.

76 P.-O. Löwdin, On the Non-Orthogonality Problem Connected with the Use of Atomic Wave Functions in the Theory of Molecules and Crystals, J. Chem. Phys., 1950, 18, 365-375.

77 W. Li, C. Ertural, D. Bogdanovski, J. Li and R. Dronskowski, Chemical Bonding of Crystalline $\mathrm{LnB}_{6}(\mathrm{Ln}=\mathrm{La}-\mathrm{Lu})$ and Its Relationship with $\mathrm{Ln}_{2} \mathrm{~B}_{8}$ Gas-Phase Complexes, Inorg. Chem., 2018, 57, 12999-13008.

Girifalco, Interaction of lithium with graphene: An ab initio study, Phys. Rev. B, 2004, 70, 125422.

79

\section{K. Rytkönen, J. Akola and M. Manninen, Density functional study} of alkali-metal atoms and monolayers on graphite (0001), Phys. Rev. B, 2007, 75, 075401.

Y. Liu, V. I. Artyukhov, M. Liu, A. R. Harutyunyan and B. I. Yakobson, Feasibility of Lithium Storage on Graphene and its Derivatives, J. Phys. Chem. Lett., 2013, 4, 1737-1742.

S. Fujikake, V. L. Deringer, T. H. Lee, M. Krynski, S. R. Elliott and G. Csányi, Gaussian approximation potential modeling of lithium intercalation in carbon nanostructures, J. Chem. Phys., 2018, 148, 241714.

M. Kühne, F. Börrnert, S. Fecher, M. Ghorbani-Asl, J. Biskupek, D. Samuelis, A. V Krasheninnikov, U. Kaiser and J. H. Smet, Reversible superdense ordering of lithium between two graphene sheets, Nature, 2018, 564, 234-239.

$\mathrm{K}$. Xu, Electrolytes and Interphases in Li-lon Batteries and Beyond, Chem. Rev., 2014, 114, 11503-11618.

J. Song, B. Xiao, Y. Lin, K. Xu and X. Li, Interphases in Sodium-Ion Batteries, Adv. Energy Mater., 2018, 8, 1703082.

N. Dupre, M. Cuisinier and D. Guyomard, Electrode/Electrolyte Interface Studies in Lithium Batteries Using NMR, Electrochem. Soc. Interface, 2016, 20, 61-67.

M. Liu, A. Kutana, Y. Liu and B. I. Yakobson, First-Principles Studies of Li Nucleation on Graphene, J. Phys. Chem. Lett., 2014, 5, 12251229.

J. M. Stratford, P. K. Allan, O. Pecher, P. A. Chater and C. P. Grey, Mechanistic insights into sodium storage in hard carbon anodes using local structure probes, Chem. Commun., 2016, 52, 1243012433.

A. P. Bartók, R. Kondor and G. Csányi, On representing chemical environments, Phys. Rev. B, 2013, 87, 184115.

S. De, A. P. Bartók, G. Csányi and M. Ceriotti, Comparing molecules and solids across structural and alchemical space, Phys. Chem. Chem. Phys., 2016, 18, 13754-13769.

M. Ceriotti, Unsupervised machine learning in atomistic simulations, between predictions and understanding, J. Chem. Phys., 2019, 150, 150901. 
